\title{
Simultaneous Sensing of Vapor Concentration and Temperature Utilizing Multimode of a MEMS Resonator
}

\author{
N. Jaber, S. Ilyas, O. Shekhah, M. Eddaoudi, and M. I. Younis \\ King Abdullah University of Science and Technology, Thuwal 23955-6900, Saudi Arabia \\ *e-mail: mohammad.younis@kaust.edu.sa
}

\begin{abstract}
Most gas sensors suffer from the cross sensitivity to environmental temperature, which significantly reduces the accuracy and reliability of measurements. Current solutions require the fabrication of a thermometer in close proximity to the gas sensor or an identical reference sensor to compensate for the sensor drift due to temperature. This increases the device size, fabrication cost, and the power required to operate the sensor; and also adds to the complexity of the device circuit for signal processing. Here, we demonstrate a single resonant gas sensor, based on a microbeam uniformly coated with metal-organic frameworks (MOFs), capable of simultaneously measuring environmental temperature and gas concentration (water vapor). Using the electrostatic harmonic voltage, we actuate the microbeam simultaneously near the first and second vibration modes. The frequency shifts of these two modes due to physical stimuli changes are monitored in real time. The lower electrode of the clamped-clamped microbeam resonator is perforated to reduce the effect of squeeze film damping, thereby allowing operation under atmospheric pressure. We demonstrate experimentally the effectiveness of this technique to measure the environmental temperature and gas concentration.
\end{abstract}

Index Terms - Multimodal, MOFs coating, electrostatic actuation, humidity and temperature sensing, and resonators.

\section{INTRODUCTION}

$\mathrm{E}$ lectrostatically actuated MEMS based resonators have been the main building block of many biological, chemical, and gas sensors. This can be attributed to the unique advantages of low power consumption, ease of fabrication, and compatibility with CMOS circuits [1]. The operation principle is based on tracking the frequency shift in the fundamental mode due to external stimuli, such as gas adsorption [2], pressure change [3], and protein detection [4]. However, the need to operate them under controlled environmental conditions and at low pressure limited their practicality [5]. Furthermore, the low-quality factor in atmospheric pressure hinders their sensitivity for gas and mass sensing applications.

In a previous work, we presented an optimized resonator design to achieve an acceptable quality factor allowing operation in air. Also, we enhanced the excitation of the higher order modes of vibration, of higher quality factor, which resulted in higher sensitivity for gas sensing [6].

Recently, significant attention has been dedicated to exploit information from the different modes of vibration of a MEMS resonator for various sensing applications, such as to extract the mechanical properties and mass of the adsorbed analyte [7], quantify the amount of attached beads or proteins on the resonator surface and locate their positions [4], weigh nanoparticles flowing inside a microfluidic channel while tracking their positions and velocities [8], and detect phase change from solid to liquid of Polyethylene Glycol [9]. Also, the coupling of two different modes has been utilized to enhance the stability and reduce fluctuation of MEMS resonators [10].

In gas sensing, the effect of environmental disturbances and the cross-sensitivity between the temperature and the amount of adsorbed gas on the sensor surface dramatically reduce the accuracy and reliability of measurements [11-13]. Several attempts have been reported in the literature to address this issue by fabricating an additional thermometer and a reference sensor in close proximity to the gas sensor. The signal from the gas sensor is corrected based on the thermometer reading [14]. Another technique is by functionalizing two identical devices with different films that have different response coefficients to temperature and humidity changes. The signals from the two devices can be utilized to quantify the variation in these physical parameters [15]. However, the need for additional physical sensors and circuits for signal postprocessing raises the fabrication cost, increases the device footprint, and adds extra demand on the power required to operate these sensors.

Thus, there is a need to develop sensitive sensors that can simultaneously sense multiple physical stimuli with the minimum power consumption. In this paper, we utilize two modes of vibrations of an electrostatically actuated MEMS resonator functionalized with metal organic frameworks (MOFs), and operated in air, to sense the change in two physical stimuli. As a case study, we demonstrate this concept to measure the temperature change and water vapor concentration. However, this concept can be extended to sense other physical parameters, such as pressure and other gas types, by optimizing the structure design to be able to actuate and track higher number of modes.

\section{FABRICATION}

The proposed sensor is composed of an electrostatically actuated clamped-clamped microbeam excited using a half electrode configuration to enhance the excitation of the second mode of vibration and functionalized with metal organic frameworks (MOFs) [16-19]. Figure 1a shows an SEM image and a schematic of the clamped-clamped resonator; of length (l) $400 \mu \mathrm{m}$, width (w) $50 \mu \mathrm{m}$, and gap (g) $3.3 \mu \mathrm{m}$. The 
formation of the MOF thin film was confirmed using X-ray diffraction (XRD) as depicted in Fig. 1b, which shows that crystalline thin film from the 10 cycles growth was obtained. The lower electrode was perforated with a perforation diameter $d=20 \mu \mathrm{m}$, and with spacing between holes $S=15 \mu \mathrm{m}$. These dimensions are selected to minimize squeeze film damping in accordance with the fabrication process rules [20]. The insets of Fig. 1a shows the uniformity of the coating over the microbeam surface.

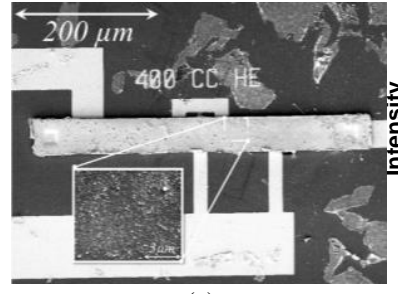

(a)

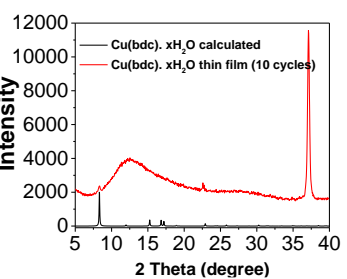

(b)

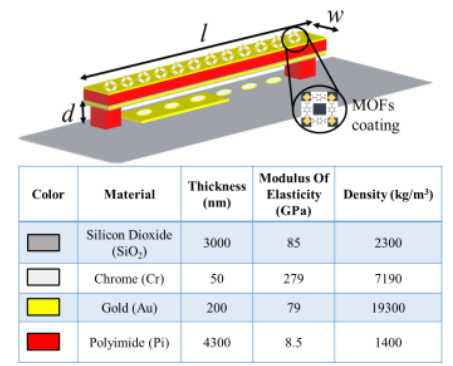

(c)

Fig. 1. (a) An SEM image of the fabricated microbeam with MOFs functionalization. (b) $\mathrm{XRD}$ of $\mathrm{Cu}(\mathrm{bdc}) \cdot \mathrm{xH}_{2} \mathrm{O} \mathrm{MOF}$ thin film grown on the microbeam (red) and its calculated pattern (black). (c) Schematic of the microbeam with the lower electrode perforation showing the material layers types, properties, and thicknesses.

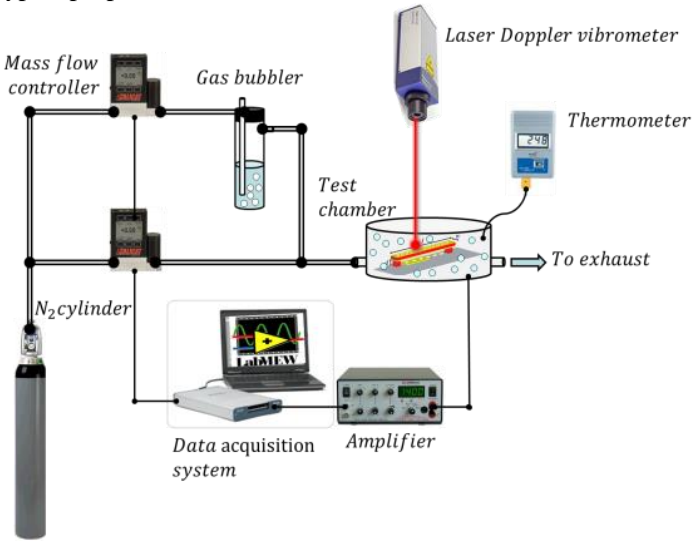

Fig. 2. Schematic of the experimental setup used for testing the device.

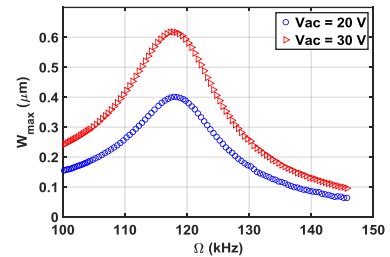

(a)

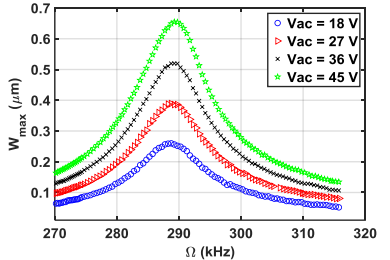

(b)
Fig. 3 Frequency response of the microbeam at $V_{D C}=50 \mathrm{~V}$ and at atmospheric chamber pressure for different AC voltages (a) near the first mode, (b) near the second mode.

\section{CHARACTERIZATION}

To characterize the microbeam, we used a Laser Doppler vibrometer to monitor the response, reveal the frequencies, mode shapes of vibration, and track the change in the amplitude of vibration due to gas exposure and temperature change. We utilized a data acquisition card (DAQ) to actuate the microbeam with different types of AC signals superimposed to a DC voltage. Also, the DAQ was used to acquire the microbeam response from the laser. Using the LabVIEW software, the recorded real time data were postprocessed to generate the frequency response curves and the frequency shift due to stimuli changes. The microbeam is placed into the test chamber, which is equipped with ports to provide the actuation signals, a photothermal heater to regulate the chamber temperature, a thermometer, and a pressure gauge. The water vapor is generated by flowing the nitrogen inside a bubbler placed in a controlled temperature bath. The vapor concentration is governed by controlling the flow of the dry nitrogen line and bubbler line. The experimental setup is depicted in Fig. 2.

\section{FREQUENCY RESPONSE}

Next, we generate the frequency response curves by exciting the microbeam with a harmonic signal while recording the maximum amplitude $W_{\max }$ of vibration, which is for the second mode is at the quarter of the microbeam length. The excitation signal is composed of an AC signal $V_{A C}$ superimposed to a DC signal $V_{D C}$. Fig. 3a and Fig. 3b show the results near the first mode and second mode of vibrations, respectively, for different $V_{A C}$ amplitudes and at atmospheric chamber pressure. These results are important to choose the suitable actuation voltage to operate the device with significant amplitudes above the noise level, and also for transforming the recorded amplitude shift due to gas exposure or temperature change into frequency data.

\section{EXPERIMENTAL RESULTS}

To experimentally extract the sensitivity of the resonator due to temperature change, we record the frequency response of the microbeam near the first and second modes at different chamber temperatures, Fig. 4. We utilized a photothermal heater to control the chamber temperature and a thermocouple placed in close proximity to the chip to record the temperature values. The experimentally extracted responsivity near the first mode is $\mathfrak{R}_{T}^{1 s t}=128 \mathrm{~Hz} /{ }^{\circ} \mathrm{C}$ and for the second mode is $\mathfrak{R}_{T}^{2 n d}=187 \mathrm{~Hz} /{ }^{\circ} \mathrm{C}$, which indicates almost 1.5 times enhanced responsivity of the second mode as reported in the insets of Fig. 4.

Figure 5 shows the real time measurements of the frequency shifts near the first and second modes of vibration at different chamber temperatures and water vapor concentrations. The real time data are recorded after flushing the chamber with Nitrogen at $1 \mathrm{l} / \mathrm{min}$ flow for an extended period of time. The water vapor concentration $C$ is controlled by changing the nitrogen flow inside the bubbler. The temperature bath is fixed at $22^{\circ} \mathrm{C}$. The vapor concentration values are calculated 
following the procedure demonstrated in [6]. It is worth to mention that a water vapor concentration of $24380 \mathrm{ppm}$ corresponds to $100 \%$ relative humidity. The responsivity of the resonator due to water vapor adsorption at $T=22^{\circ} \mathrm{C}$ near the first and second modes are $\mathfrak{R}_{C}^{1 s t}=0.68 \mathrm{~Hz} / \mathrm{ppm}$ and $\mathfrak{R}_{C}^{2 n d}=1.2 \mathrm{~Hz} / \mathrm{ppm}$, respectively. The minimum detectable concentration is $\Delta C_{\min }=\Delta f_{\min } / \mathfrak{R}_{C}^{2 n d}=4.6 p p m . \quad$ As expected, a better sensitivity is demonstrated near the second mode. The improved sensitivity near the second mode encourages the effort toward optimized resonator designs to efficiently excite the higher order modes of vibration for ultrasensitive applications.

By investigating the real time data shown in Fig. 5a, Fig. $5 \mathrm{~b}$, and Fig. 5c, we note that increasing the temperature at particular concentration values decreases the recorded frequency shift due to the reduced amount of water molecules adsorbed on the resonator surface. The same conclusion can be drawn from Fig. $5 \mathrm{~b}$ and Fig. 5c, where at higher concentration the frequency shifts at $22^{\circ} \mathrm{C}$ approach the measured values at $38^{\circ} \mathrm{C}$ for the first mode, and exceed them for the second mode.

Theoretically, the frequency ratio $R$ depends only on the temperature change and is independent of the amount of mass being adsorbed on the resonator surface. Hence, the frequency ratio are expected to be horizontal lines and does not depend on the water vapor concentration. However, the experimentally recorded ratios, Fig. 5f, show a slight deviation, which can be attributed to the temperature fluctuation, and the different vibrational velocity of each point along the beam length. From basic adsorption theories, it is known that gas adsorption strongly depends on the surface temperature, gas concentration, and the velocity of the surface. Hence, even if the MOFs coating was uniformly distributed along the beam length, the difference in vibrational velocity along the beam results in position-dependent adsorption. To verify this, future experiments should be designed and conducted to quantify the velocity effect on adsorption.

The significance of the presented technique can be demonstrated by considering a case study where the experimentally measured frequency values near the first and second modes are $117.8 \mathrm{kHz}$ and $290 \mathrm{kHz}$, respectively. The corresponding frequency ratio $R$ is 2.461 . The case study data are highlighted with black arrow in Fig. 5d, Fig. 5e, and Fig. 5f. From the arrow intersection with the different curves, one can note that the only point that satisfies the measured frequency values and their corresponding ratio is $1750 \mathrm{ppm}$ concentration at $45^{\circ} \mathrm{C}$. Hence, using two modes of vibration of a single microbeam, it is possible to measure the change in two physical stimuli. These findings can be potentially extended to measure multiple physical parameters by increasing the number of modes.

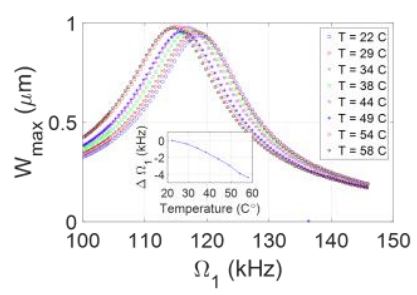

(a)

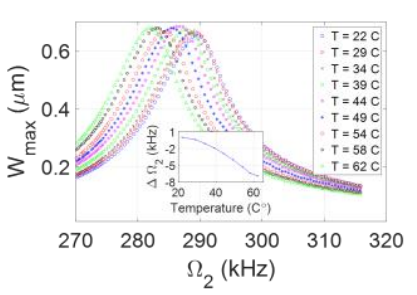

(b)
Fig. 4 Frequency response curves at $V_{D C}=50 \mathrm{~V}$, atmospheric pressure, and for different chamber temperature, (a) near the first mode at $V_{A C}=30 \mathrm{~V}$, (b) near the second mode at $V_{A C}=36 \mathrm{~V}$. Inset: the corresponding resonance frequency for each temperature value.

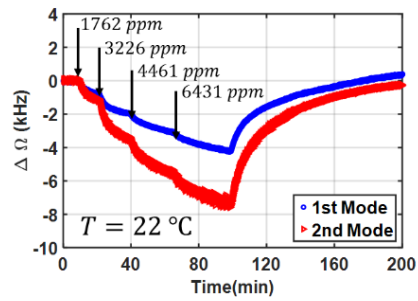

(a)

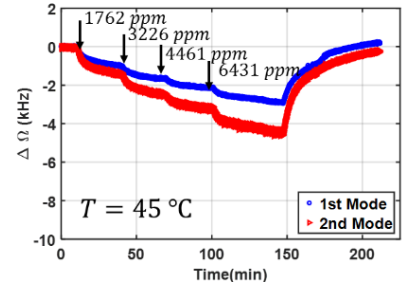

(c)

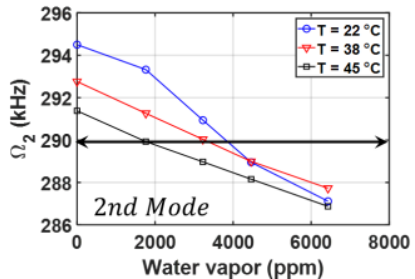

(e)

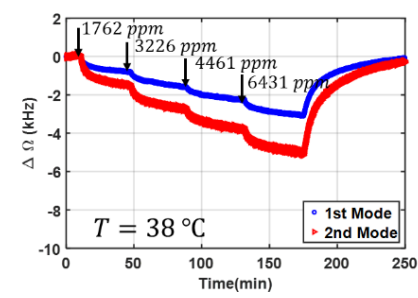

(b)

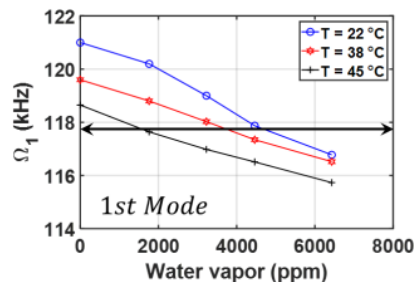

(d)

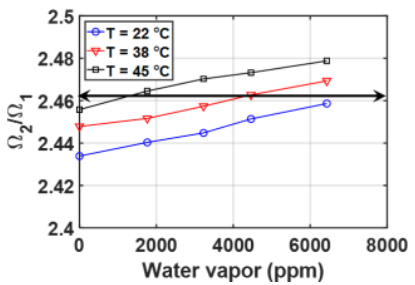

(f)
Fig. 5. Experimentally measured frequency shifts near the first and second mode of vibration for different water vapor concentrations at chamber temperature of (a) $22{ }^{\circ} \mathrm{C}$, (b) $38{ }^{\circ} \mathrm{C}$, and (c) $45{ }^{\circ} \mathrm{C}$. Summary of the experimentally measured frequency shifts at different combinations of water vapor concentration and chamber temperature near (d) the first mode, (e) the second mode, and (f) the two frequencies ratio.

\section{CONCLUSIONS}

We presented a new technique to measure two physical stimuli using a single electrostatically actuated resonant microbeam coated with MOFs and operated in air. The method is based on simultaneously actuating and tracking two frequencies of two vibration modes of a microstructure. As a case study, we utilized this concept to measure the environmental temperature and humidity concentration. Optimizing the resonator design to excite higher order modes will open the door for simultaneously measuring multiple physical stimuli using a single resonator, which reduces the power consumption, device size, and cost, and leads to smarter generation of sensors.

\section{ACKNOWLEDGEMENTS}

We acknowledge financial support from King Abdullah University of Science and Technology. 


\section{REFERENCES}

[1] Younis, M. I. MEMS Linear and Nonlinear Statics and Dynamics, (Springer, 2011).

[2] Yamagiwa, H. et al. Detection of Volatile Organic Compounds by Weight-Detectable Sensors coated with Metal-Organic Frameworks. Scientific Reports 4, 164 -168 (2014).

[3] Hajjaj, A. Z., Alcheikh, N., Hafiz, M. A. A., Ilyas, S. \& Younis, M. I. A scalable pressure sensor based on an electrothermally and electrostatically operated resonator. Applied Physics Letters 111, 223503, (2017).

[4] Hanay, M. S. et al. Single-protein nanomechanical mass spectrometry in real time. Nature Nanotechnology 7, 602-608 (2012).

[5] Xu, Y., Lin, J. T., Alphenaar, B. W. \& Keynton, R. S. Viscous damping of microresonators for gas composition analysis. Applied Physics Letters 88, 143513, (2006).

[6] Jaber, N., Ilyas, S., Shekhah, O., Eddaoudi, M. \& Younis, M. I. Resonant Gas Sensor and Switch Operating in Air With Metal-Organic Frameworks Coating. Journal of Microelectromechanical Systems 27, 156163 (2018).

[7] Gil-Santos, E. et al. Nanomechanical mass sensing and stiffness spectrometry based on two-dimensional vibrations of resonant nanowires. Nature Nanotechnology 5, 641-645 (2010).

[8] Olcum, S., Cermak, N., Wasserman, S. C. \& Manalis, S. R. High-speed multiple-mode mass-sensing resolves dynamic nanoscale mass distributions. Nature Communications 6, 7070, (2015).

[9] Prasad, A. \& Seshia, A. A. Detection of phase transition in polyethylene glycol using a multimodal micromechanical acoustic resonator. Applied Physics Letters 110, 134101, (2017).

[10] Antonio, D., Zanette, D. H. \& López, D. Frequency stabilization in nonlinear micromechanical oscillators. Nature Communications 3, 806, (2012).

[11] Assen, A. H., Yassine, O., Shekhah, O., Eddaoudi, M. \& Salama, K. N MOFs for the Sensitive Detection of Ammonia: Deployment of fcu-MOF Thin Films as Effective Chemical Capacitive Sensors. ACS Sensors 2, 12941301 (2017)

[12] Xu, P., Yu, H., Guo, S. \& Li, X. Microgravimetric Thermodynamic Modeling for Optimization of Chemical Sensing Nanomaterials. Analytical Chemistry 86, 4178-4187 (2014).

[13] Lv, Y., Yu, H., Xu, P., Xu, J. \& Li, X. Metal organic framework of MOF-5 with hierarchical nanopores as micro-gravimetric sensing material for aniline detection. Sensors and Actuators B: Chemical 256, 639-647 (2017)

[14] Oprea, A. et al. Temperature, humidity and gas sensors integrated on plastic foil for low power applications. Sensors and Actuators B: Chemical 140, 227-232 (2009).

[15] Leng, X., Li, W., Luo, D. \& Wang, F. Differential Structure With Graphene Oxide for Both Humidity and Temperature Sensing. IEEE Sensors Journal 17, 4357-4364 (2017).

[16] Yassine, O. et al. H2S Sensors: Fumarate-Based fcu-MOF Thin Film Grown on a Capacitive Interdigitated Electrode. Angewandte Chemie International Edition 55, 15879-15883 (2016).

[17] Yim, C. et al. CO2-Selective Nanoporous Metal-Organic Framework Microcantilevers. Scientific Reports 5, 10674 (2015).

[18] Sapsanis, C. et al. Insights on Capacitive Interdigitated Electrodes Coated with MOF Thin Films: Humidity and VOCs Sensing as a Case Study. Sensors 15, 18153-18166 (2015).
[19] Azzam, E. M. S. et al. Fabrication of a surface plasmon resonance biosensor based on gold nanoparticles chemisorbed onto a 1,10-decanedithiol self-assembled monolayer. Thin Solid Films 518, 387-391 (2009).

[20] Arevalo, A. et al. A versatile multi-user polyimide surface micromachinning process for MEMS applications in IEEE 10th International Conference on Nano/Micro Engineered and Molecular Systems (NEMS),561565 (2015). 